\title{
A comparative study of rigid Earth, non-rigid Earth nutation theories, and observational data
}

\author{
J. Souchay ${ }^{1}$, S. B. Lambert ${ }^{2}$, and C. Le Poncin-Lafitte ${ }^{3}$ \\ 1 Observatoire de Paris, SYRTE, CNRS/UMR8630, 75014 Paris, France \\ e-mail: jean. souchay@obspm.fr \\ 2 Royal Observatory of Belgium, 1180 Brussels, Belgium \\ e-mail: s. lambert@oma.be \\ 3 Lohrmann Observatory, Dresden Technical University, 01062 Dresden, Germany \\ e-mail: christophe.le_poncin-lafitte@tu-dresden.de
}

Received 8 January 2007 / Accepted 20 March 2007

\section{ABSTRACT}

\begin{abstract}
Context. The past decade has shown strong development of non-rigid Earth nutation theories at the microarcsecond level, now able to match the VLBI observations with differences of about $200 \mu$ as in rms, leading the IAU to adopt the conventional analytical model MHB 2000 (Mathews et al. 2002, J. Geophys. Res., 107, B4). This model has been established starting from the REN 2000 rigid Earth nutation theory (Souchay et al. 1999, A\&AS, 135, 111).

Aims. Users generally do not know the differences between each coefficient of the Fourier series in MHB 2000 and the corresponding one in REN 2000. The aim of this paper is to clarify these differences.

Methods. After recalling how the nutation coefficients were obtained in the two above theories, we catch the coefficients that are particularly affected by the non-rigidity. Beside, we develop statistics on the coefficients that are not influenced.

Results. At the same time we show some anomalies concerning some coefficients present in REN 2000, but not in MHB 2000 and inversely. The influence of those lacking coefficients is evaluated. Then we simutaneously calculate the REN 2000 and MHB 2000 nutations, thus showing the global contribution of the non-rigidity of the Earth on the nutation. We compare this difference against the differences between observational data and MHB 2000.
\end{abstract}

Key words. celestial mechanics - astrometry - reference systems - Earth

\section{Introduction}

Nutation describes the motion of the Earth's figure axis with respect to a space-fixed frame of reference. It is one of the three components of the coordinate transformation between the terrestrial and the celestial frames, together with the polar motion and the Earth's rotation angle (UT1). Accurate knowledge of the nutation is important for geophysics, precise positioning, space navigation, or astrometric data reduction. Very long baseline radio interferometry (VLBI) or lunar laser ranging (LLR) give access to the nutation angles. Global navigation and satellite system (GNSS) measurements can also help for getting their time derivatives. But only VLBI provides very accurate nutation time series for more than 25 years on a very regular basis. During the past decade, technical developments (data acquisition systems, extension of the network) and improvements in modeling and analysis strategies brought the precision of the routine VLBI observations down to the sub milliarc second (mas) level. In the frequency domain, the coefficients of the nutation spectral components can be determined with an accuracy running from a hundred microarc seconds ( $\mu$ as) for long periods down to a few tens of $\mu$ as for shorter periods.

A recent challenge concerning nutation consists of constructing an analytical theory at best matching the observational data. A new nutation series called MHB 2000 (Mathews et al. 2002, referred to as MHB in the following) was adopted at the IAU XXIVth General Assembly held in Manchester, UK. It was obtained by direct solution of the linearized equations of the non rigid Earth's rotation, with best-fit values for various geophysical parameters adjusted on VLBI data. It leads to a considerably better match to observational data than any of the earlier series based on geophysical models (e.g., Wahr 1981): VLBI observations against the MHB model show differences of less than $200 \mu$ as in rms.

Forced nutation is entirely due to the gravitational torques exerted by the external bodies (the Moon, the Sun, but also the planets whose effects cannot be neglected anymore). In order to build the MHB series, Mathews et al. (2002) started from a rigid Earth nutation series representing the action of the external gravitational potential on a hypothetical rigid Earth having the same physical characteristics (mass, harmonic coefficients of the geopotential) as the real Earth. During the past decade, the precision of the rigid Earth's nutation spectral coefficients has increased by two orders of magnitude. While the series of Kinoshita \& Souchay (1990) were calculated with a 0.01 mas truncation level, the REN 2000 series (referred to as REN in the following) are tabulated with a $0.1 \mu$ as truncation level. This made it possible to use the observed nutation data sets to estimate the amplitudes of a drastically larger number of nutation 
coefficients with uncertainties that are about an order of magnitude smaller than at the beginning of the 1990s.

For the common user of nutation series, as well as for the Earth's rotation specialist, differences between rigid and nonrigid Earth nutation is sometimes not perfectly understood, both qualitatively and quantitatively. This paper is devoted to a complete and detailed analysis of these differences. The contribution of non-rigidity in the global nutation signal is clarified. We also evaluate the influence of the coefficients of nutation with very long periods. Moreover, we point out the presence of some specific nutation terms present in MHB and not in REN, and reciprocally. Finally we calculate the impact of the number of coefficients taken into account in the analytical nutation.

\section{Rigid and non-rigid Earth nutations series}

\subsection{Overview of REN and $M H B$}

The REN tables of the nutation for a rigid Earth (Souchay et al. 1999) were obtained starting from a Hamiltonian theory first set up by Kinoshita $(1972,1977)$. They include a lot of contributions neglected or partly considered in previous tables (Kinoshita 1977; Kinoshita \& Souchay 1990). These new contributions are the indirect planetary effects contained in the lunisolar perturbing function (Souchay \& Kinoshita 1996), as well as the direct planetary contributions (Souchay \& Kinoshita 1997), together with second-order terms coming from crossed nutations and spin-orbit couplings when considering the Earth-Moon system as a whole. Moreover they take the influence of zonal geopotential harmonics of the Earth $\left(J_{3}, J_{4}\right)$ into account. Quasi-diurnal and quasi semi-diurnal components coming from the harmonics of degree 2, 3, and 4 (Folgueira et al. 1998, 1999) of the geopotential were also added to the series. One of the by-products of the series REN was a complete expression of the general precession in longitude $\psi_{A}$ with its various contributions (Souchay et al. 1996).

In parallel with these works, two concurrent theories of a rigid Earth nutation were set up: SMART97 (Bretagnon et al. 1997) and RDAN97 (Roosbeek \& Dehant 1998). In order to validate the quality of REN, Souchay (1998) compared it against the above-mentioned theories. He showed that no significant differences exist between these works at the level of a few $\mu$ as, except for the 18.6-yr component, after fitting the dynamical flattening. The agreement was still better when he compared the nutation with what was obtained from a numerical integration of the dynamical equations. All these various checks justify that REN, with its coefficients truncated at $0.1 \mu$ as, looks valid at the $\mu$ as level. Note that each of the three nutation series above give the forced nutation for each: the angular momentum axis, the rotation axis and the figure axis.

The MHB nutation series for a non-rigid Earth (Mathews et al. 2002) are the results of numerous efforts understanding various effects dealing with the geophysical response of our planet to the external torque exerted by the Moon, the Sun, and the planets. The authors modified the dynamical equations developed in a previous work (Mathews et al. 1991) by including several other contributions. First, the effects of anelasticity are taken into account through complex and frequency dependent compliances (deformability parameters). Another improvement is the construction of an empirical ocean loading and of current admittance functions for the purpose. The MHB theory also includes the effects of electromagnetic coupling at the core and inner-core boundaries and of non linear terms (a more complete computation of the non linear terms is found in
Lambert \& Mathews 2006). Taking as a basis the REN series for a rigid Earth, MHB expressed the ratio between the non-rigid nutation spectral coefficients and their rigid counterparts as a (transfer) function depending upon the frequency and a small number of parameters including the complex eigenfrequencies of the three-layer Earth's free rotational modes and the ellipticity. Thus one can build the MHB series by applying this transfer function to the REN series and then adding on the various contributions not directly related to non-rigidity (listed in Table 7 of the MHB paper).

Note that in the following we separate the nutation in longitude $\Delta \psi$ and the nutation in obliquity $\Delta \epsilon$, since it is the way they are expressed in the REN and MHB tables and in a large part of the literature. Nevertheless, the MHB complex transfer function is to be applied to prograde and retrograde nutation amplitudes, which are given by an appropriate complex combination of $\Delta \psi$ and $\Delta \epsilon$.

\subsection{Characteristics of the original REN}

The REN nutation series for the axis of figure of the Earth are presented for both $\Delta \psi$ and $\Delta \epsilon$ as the sum of sinusoidal terms, each of them the sum of the sine and a cosine terms whose phase is a linear combination of the 5 Delaunay arguments $\left(l, l^{\prime}, F, D, \Omega\right)$ and of the mean longitudes of the planets $\lambda_{\mathrm{Me}}, \lambda_{\mathrm{Ve}}, \lambda_{\mathrm{Ea}}$ etc. A last column indicates the linear evolution of the sine amplitude for $\Delta \psi$ and of the cosine for $\Delta \epsilon$. The series in their final form are sorted by decreasing period. A flag indicates the origin of the coefficients:

M1: Main Problem (Moon),

S: Sun,

PS: Planetary Sun,

PM: Planetary Moon,

T2: Terms with second-order contributions,

J3: Terms coming from the $J_{3}$ zonal harmonic potential of the Earth,

ME, VE, MA, JU, SA, UR, NE: Direct action of the planets. The second-order contributions in the terms quoted "T2" are generally much smaller than the first-order ones obtained by direct application of the canonical equations in the theory (Kinoshita 1977; Kinoshita \& Souchay 1990). They come from two different origins. The first one is the crossed nutation effects, where the effect of the nutation modifies the true equatorial plane so that it is different from the mean plane subject only to the precession. This difference creates an additional effect on nutation components which is only significant for the largest coefficients. The second origin is the spin-orbit coupling: the $J_{2}$ coefficient of the geopotential, characterizing the flattening of the Earth, influences the Moon's orbit, which in turn modifies the lunar potential and consequently the lunar torque exerted on the Earth. The calculation of this contribution in Hamiltonian theory needs a complete theoretical development with considering the EarthMoon system as a whole. Here also, only the largest nutation terms are significantly affected by this effect.

\subsection{Construction of a combined REN}

For clear knowledge of the origin of a given coefficient, Souchay et al. (1999) did not gather as a single coefficient those that have the same arguments but rather different origins as specified in the preceding section. The purpose in this section is to give information about these coefficients and to transform the original 
series into a combined series in which only one term exists for a given frequency.

Concerning $\Delta \psi$, REN originally contains 1482 terms. Among them, 1246 are single, meaning that they cannot be coupled with other terms (because they do not have the same argument as any of the other ones). But 194 are double (can be associated with another term) and 42 are triple (can be associated with two other terms). Therefore instead of the original series containing 1482 terms, a combined series can be constructed with only $1357(1246+97+14)$ terms. Concerning $\Delta \epsilon$, REN contains 1118 terms in its original form. Among them 63 are double and only one is triple. Then the combined $\Delta \epsilon$ series contains 1053 terms $(989+63+1)$.

Although it should be exhaustive to present all the combined coefficients (double or triple) in a table, we decided to show only the triple ones in Table 1 in order to show how a single nutation coefficient can be affected by various contributions.

\section{Comparisons between REN and MHB}

As the MHB series have been constructed starting from the REN series, it looks interesting to carry out a term-per-term association, which means that we identify each component of one of the series from its argument, and we search the corresponding coefficient of the other series with the same argument. In the general case of identification, we make a systematic study of the differences between the amplitudes (due to the non rigidity of the Earth). Moreover we point out the cases of non identification (lacking terms when making the correspondence between the two series).

\subsection{Inconsistencies between the two series}

Concerning $\Delta \psi$, MHB contains 1320 coefficients that have to be compared with the 1357 coefficients present in the combined REN. The counts are 1037 coefficients and 1053 coefficients for $\Delta \epsilon$. The number of coefficients with common arguments in both series are 1304 for $\Delta \psi$ and 1014 for $\Delta \epsilon$.

When looking at these differences in more details 16 coefficients for $\Delta \psi$ with arguments present in MHB are not found in REN, among which 2 correspond to coefficients with an amplitude larger than $10 \mu$ as and 7 with an amplitude between $1 \mu$ as and $10 \mu$ as. These 9 largest coefficients are listed in Table 2. For $\Delta \epsilon, 23$ coefficients with arguments present in MHB are not found in REN, none having an amplitude larger than $10 \mu \mathrm{as}$, whereas 11 coefficients have amplitudes between $1 \mu$ as and $10 \mu$ as. These largest coefficents are listed in Table 3.

Reciprocally, $53 \Delta \psi$ coefficients present in REN are not found in MHB, among which 3 have amplitudes larger than $10 \mu$ as, and 11 have amplitudes between $1 \mu$ as and $10 \mu$ as. These largest coefficients are listed in Table 4 . For $\Delta \epsilon, 39$ coefficients present in REN are not included in MHB, among which 9 have amplitudes between $1 \mu$ as and $10 \mu$ as. They are listed in Table 5 .

It looks obvious that, for most of the terms listed in Tables 2-5, the amplitudes are the same in MHB and REN, but with different arguments. This suggests that for these terms some errors could have been made in the arguments when constructing MHB from REN. This is for instance the case of the terms with amplitudes $2.1 \mu$ as (sine) and $1.1 \mu$ as (cosine), or with amplitudes $2.4 \mu$ as (sine) and $1.2 \mu$ as (cosine) in Table 2, which must undoubtedly be associated with the terms with these same amplitudes in Table 4. Note that in REN a lot of terms are coupled according to close frequencies, like the terms with period
Table 1. Coefficients of nutation in the series of nutation for a rigid Earth model REN in $\Delta \psi$ with three different contributions.

\begin{tabular}{|c|c|c|c|c|}
\hline Argument & $\begin{array}{r}\text { Period } \\
\text { days }\end{array}$ & $\begin{array}{l}\text { Sine } \\
\text { mas }\end{array}$ & $\begin{array}{r}\text { Cosine } \\
\text { mas }\end{array}$ & Origin \\
\hline $2 \lambda_{\mathrm{Ju}}-5 \lambda_{\mathrm{Sa}}$ & -322614.513 & -0.0487 & 0.0113 & PS \\
\hline- & - & -0.0004 & 0.0000 & SA \\
\hline \multirow[t]{2}{*}{-} & - & 0.0000 & 0.0015 & JU \\
\hline & - & -0.0491 & 0.0128 & TOTAL \\
\hline$\lambda_{\mathrm{Ea}}-2 \lambda_{\mathrm{Ma}}$ & -5764.006 & -0.0368 & 0.0387 & PS \\
\hline- & - & -0.0088 & 0.0050 & MA \\
\hline \multirow[t]{2}{*}{ - } & - & 0.0007 & -0.0007 & PM \\
\hline & - & -0.0449 & 0.0430 & TOTAL \\
\hline $2 \lambda_{\mathrm{Ju}}+2 p_{\mathrm{A}}$ & 5373.471 & -0.0123 & 0.0000 & SA \\
\hline- & - & -0.0014 & 0.0000 & PS \\
\hline \multirow[t]{2}{*}{-} & - & 0.0004 & 0.0015 & JU \\
\hline & - & -0.0133 & 0.0000 & TOTAL \\
\hline$\lambda_{\mathrm{Ju}}$ & 4332.589 & -0.0425 & 0.0299 & PS \\
\hline- & - & 0.0334 & -0.0047 & $\mathrm{JU}$ \\
\hline \multirow[t]{2}{*}{-} & - & 0.0000 & -0.0004 & PM \\
\hline & - & -0.0091 & 0.0248 & TOTAL \\
\hline $2 \lambda_{\mathrm{Ve}}-3 \lambda_{\mathrm{Ea}}$ & 1454.936 & 0.0008 & 0.0485 & PS \\
\hline- & - & 0.0000 & -0.0009 & PM \\
\hline \multirow[t]{2}{*}{-} & - & 0.0000 & 0.0138 & VE \\
\hline & - & 0.0008 & 0.0614 & TOTAL \\
\hline$\lambda_{\mathrm{Ve}}-\lambda_{\mathrm{Ea}}$ & 583.921 & -0.0022 & 0.0000 & $\mathrm{PM}$ \\
\hline- & - & 0.0846 & 0.0000 & VE \\
\hline \multirow[t]{2}{*}{-} & - & 0.0661 & 0.0000 & PS \\
\hline & - & -0.0491 & 0.0128 & TOTAL \\
\hline$\lambda_{\mathrm{Ea}}-2 \lambda_{\mathrm{Ju}}$ & 439.332 & -0.0185 & 0.0270 & PS \\
\hline- & - & 0.0019 & 0.0003 & $\mathrm{JU}$ \\
\hline \multirow[t]{2}{*}{-} & - & 0.0000 & -0.0004 & PM \\
\hline & - & -0.0166 & 0.0269 & TOTAL \\
\hline $3 \lambda_{\mathrm{Ve}}-4 \lambda_{\mathrm{Ea}}$ & 416.688 & -0.0005 & 0.0293 & PS \\
\hline- & - & 0.0000 & -0.0004 & PM \\
\hline \multirow[t]{2}{*}{-} & - & 0.0000 & -0.0039 & VE \\
\hline & - & -0.0005 & 0.0250 & TOTAL \\
\hline$\lambda_{\mathrm{Ea}}-\lambda_{\mathrm{Ju}}$ & 398.884 & -0.1355 & -0.0026 & PS \\
\hline- & - & 0.0116 & 0.0000 & JU \\
\hline \multirow[t]{2}{*}{-} & - & 0.0016 & 0.0000 & PM \\
\hline & - & -0.1223 & -0.0026 & TOTAL \\
\hline $2 \lambda_{\mathrm{Ea}}-2 \lambda_{\mathrm{Ma}}$ & 389.968 & -0.0385 & 0.0000 & PS \\
\hline- & - & 0.0012 & 0.0000 & JU \\
\hline \multirow[t]{2}{*}{-} & - & 0.0005 & -0.0000 & PM \\
\hline & - & -0.0367 & 0.0000 & TOTAL \\
\hline$\lambda_{\mathrm{Ea}}$ & 365.256 & 0.0005 & 0.0000 & JU \\
\hline- & - & 0.0003 & 0.0000 & MA \\
\hline \multirow[t]{2}{*}{-} & - & 0.0000 & -0.0027 & VE \\
\hline & - & 0.0008 & -0.0027 & TOTAL \\
\hline $2 \lambda_{\mathrm{Ve}}-2 \lambda_{\mathrm{Ea}}$ & 291.961 & -0.0956 & 0.0000 & PS \\
\hline- & - & 0.0350 & 0.0000 & VE \\
\hline \multirow[t]{2}{*}{-} & - & 0.0008 & 0.0000 & JU \\
\hline & - & -0.0598 & 0.0000 & TOTAL \\
\hline \multirow{4}{*}{$\begin{array}{l}2 \lambda_{\mathrm{Ea}}-2 \lambda_{\mathrm{Ju}} \\
- \\
-\end{array}$} & 199.442 & -0.0010 & 0.0000 & PM \\
\hline & - & 0.0389 & 0.0003 & PS \\
\hline & - & 0.0014 & 0.0000 & JU \\
\hline & - & 0.0293 & 0.0003 & TOTAL \\
\hline \multirow{4}{*}{$\begin{array}{l}3 \lambda_{\mathrm{Ve}}-3 \lambda_{\mathrm{Ea}} \\
- \\
-\end{array}$} & 194.640 & -0.0094 & 0.0000 & PS \\
\hline & - & -0.0004 & 0.0000 & PM \\
\hline & - & 0.0187 & 0.0000 & VE \\
\hline & - & 0.0089 & 0.0000 & TOTAL \\
\hline
\end{tabular}

$13.635 \mathrm{~d}$ and $13.632 \mathrm{~d}$ or $9.134 \mathrm{~d}$ and $9.132 \mathrm{~d}$ (Table 4). The reason the corresponding coefficients must have the same amplitudes is easily explained by the fact that they result from the multiplication of two sinusoidal oscillations of frequencies $\omega_{1}$ and $\omega_{2}$ with $\omega_{2} \ll \omega_{1}$, which can be transformed into two 
Table 2. Coefficients of nutation $\Delta \psi$ with arguments present in MHB series and not in REN.

\begin{tabular}{lrrr}
\hline \hline Argument & $\begin{array}{r}\text { Period } \\
\text { days }\end{array}$ & $\begin{array}{r}\text { Sine } \\
\mu \text { as }\end{array}$ & $\begin{array}{r}\text { Cosine } \\
\mu \text { as }\end{array}$ \\
\hline $6 \lambda_{\mathrm{Ea}}$ & 60.876 & 1.4 & 0.0 \\
$11 \lambda_{\mathrm{Ea}}$ & 33.203 & -1.1 & 0.5 \\
$l-2 F-2 \Omega+4 \lambda_{\mathrm{Ea}}-8 \lambda_{\mathrm{Ma}}+3 \lambda_{\mathrm{Ju}}$ & 27.091 & 12.6 & -6.3 \\
$l-2 F-\Omega-\lambda_{\mathrm{Ea}}$ & 25.128 & 2.1 & -1.1 \\
$2 l-2 \lambda_{\mathrm{Ea}}+3 \lambda_{\mathrm{Ju}}$ & 14.749 & -2.1 & 1.1 \\
$2 F+2 \Omega+\lambda_{\mathrm{Ea}}$ & 13.168 & -2.4 & -1.2 \\
$l+2 F+\Omega$ & 9.357 & 2.1 & -1.1 \\
$l+2 F+2 \Omega+\lambda_{\mathrm{Ea}}$ & 8.910 & 13.1 & -6.3 \\
$2 l+2 F+2 \Omega+2 \lambda_{\mathrm{Ea}}-3 \lambda_{\mathrm{Ju}}$ & 6.641 & 2.4 & -1.2 \\
\hline
\end{tabular}

Table 3. Coefficients of nutation $\Delta \epsilon$ with arguments present in MHB series and not present in REN.

\begin{tabular}{lrrr}
\hline \hline Argument & $\begin{array}{r}\text { Period } \\
\text { days }\end{array}$ & $\begin{array}{r}\text { Sine } \\
\mu \text { as }\end{array}$ & $\begin{array}{r}\text { Cosine } \\
\mu \text { as }\end{array}$ \\
\hline $2 l-l^{\prime}-2 \Omega$ & 471.891 & 0.2 & 1.2 \\
$l^{\prime}-2 F+2 \Omega$ & 329.791 & 0.0 & 2.9 \\
$2 \lambda_{\mathrm{E}}$ & 182.628 & 0.0 & -3.5 \\
$2 \lambda_{\mathrm{Ve}}+\lambda_{\mathrm{Ea}}+p_{\mathrm{A}}$ & 85.921 & 0.2 & -1.0 \\
$l-2 F-2 \Omega+4 \lambda_{\mathrm{E}}-8 \lambda_{\mathrm{Ma}}+3 \lambda_{\mathrm{Ju}}$ & 27.091 & 2.7 & 5.5 \\
$l-2 F-\Omega-\lambda_{\mathrm{Ea}}$ & 25.128 & 0.6 & 1.1 \\
$2 l-2 \lambda_{\mathrm{E}}+3 \lambda_{\mathrm{Ju}}$ & 14.749 & -0.5 & 1.0 \\
$2 F+2 \Omega+\lambda_{\mathrm{E}}$ & 13.168 & -2.4 & -1.2 \\
$l+2 F+\Omega$ & 9.357 & -0.6 & -1.1 \\
$l+2 F+2 \Omega+\lambda_{\mathrm{Ea}}$ & 8.910 & -2.6 & -5.7 \\
$2 l+2 F+2 \Omega+2 \lambda_{\mathrm{Ea}}-3 \lambda_{\mathrm{Ju}}$ & 6.641 & -0.5 & -1.1 \\
\hline
\end{tabular}

Table 4. Coefficients of nutation $\Delta \psi$ with arguments present in the REN series and not in MHB.

\begin{tabular}{lrrr}
\hline \hline Argument & $\begin{array}{r}\text { Period } \\
\text { days }\end{array}$ & $\begin{array}{r}\text { Sine } \\
\mu \text { as }\end{array}$ & $\begin{array}{r}\text { Cosine } \\
\mu \text { as }\end{array}$ \\
\hline $10 \lambda_{\mathrm{Ea}}-16 \lambda_{\mathrm{Ma}}+4 \lambda_{\mathrm{JU}}+5 \lambda_{\mathrm{Sa}}+2 p_{\mathrm{A}}$ & 182.620 & -1.3 & -4.3 \\
$8 \lambda_{\mathrm{Ve}}-11 \lambda_{\mathrm{Ea}}+2 p_{\mathrm{A}}$ & 182.240 & -6.2 & -9.7 \\
$-10 \lambda_{\mathrm{Ve}}+12 \lambda_{\mathrm{Ea}}+2 p_{\mathrm{A}}$ & 85.839 & 2.4 & 0.0 \\
$-11 \lambda_{\mathrm{Ve}}+13 \lambda_{\mathrm{Ea}}+2 p_{\mathrm{A}}$ & 74.837 & 1.7 & 0.0 \\
$-2 l+18 \lambda_{\mathrm{V}}-16 \lambda_{\mathrm{Ea}}$ & 27.562 & 2.1 & 1.1 \\
$l+2 F+2 \Omega-18 \lambda_{\mathrm{Ve}}+16 \lambda_{\mathrm{Ea}}$ & 13.663 & 12.6 & -6.3 \\
$-l+2 F+2 \Omega+18 \lambda_{\mathrm{Ve}}-16 \lambda_{\mathrm{Ea}}$ & 13.659 & -12.6 & -6.3 \\
$l+2 F+\Omega-18 \lambda_{\mathrm{Ve}}+16 \lambda_{\mathrm{Ea}}$ & 13.635 & 2.1 & -1.1 \\
$-l+2 F+\Omega+18 \lambda_{\mathrm{Ve}}-16 \lambda_{\mathrm{Ea}}$ & 13.632 & -2.1 & -1.1 \\
$2 l+2 F+2 \Omega-18 \lambda_{\mathrm{Ve}}+16 \lambda_{\mathrm{Ea}}$ & 9.134 & 2.4 & -1.2 \\
$2 F+2 \Omega+18 \lambda_{\mathrm{Ve}}-16 \lambda_{\mathrm{Ea}}$ & 9.132 & -2.4 & -1.2 \\
\hline
\end{tabular}

oscillations with respective frequencies $\omega_{1}+\omega_{2}$ and $\omega_{1}-\omega_{2}$ both very close to $\omega_{1}$. Therefore after integration (i.e., division by the frequency), the amplitudes of these terms still remain very close.

The global rms of the signal obtained for a 10-yr time span by summing the components listed in Tables 2-5 are $14.9 \mu$ as, $6.8 \mu \mathrm{as}, 17.2 \mu \mathrm{as}$, and $7.5 \mu \mathrm{as}$, respectively.

\subsection{Coefficients with common arguments in the two series}

Table 6 shows the number of coefficients per interval of amplitude when REN and MHB are restricted to their common terms (1304 for $\Delta \psi$ and 1014 for $\Delta \epsilon$ ). We can observe that the increase in the number of coefficients steadily ranges between 2.5 and 3 when we turn from a given interval of amplitude to the following one, except for the first and last intervals. Moreover, in order to measure the influence of the non-rigidity of the Earth
Table 5. Coefficients of nutation $\Delta \epsilon$ with arguments present in the REN series and not in MHB.

\begin{tabular}{lrrr}
\hline \hline Argument & $\begin{array}{r}\text { Period } \\
\text { days }\end{array}$ & $\begin{array}{r}\text { Sine } \\
\mu \text { as }\end{array}$ & $\begin{array}{r}\text { Cosine } \\
\mu \text { as }\end{array}$ \\
\hline $10 \lambda_{\mathrm{Ea}}-16 \lambda_{\mathrm{Ma}}+4 \lambda_{\mathrm{JU}}+5 \lambda_{\mathrm{Sa}}+2 p_{\mathrm{A}}$ & 182.620 & -1.9 & 0.6 \\
$10 \lambda_{\mathrm{Ve}}-11 \lambda_{\mathrm{Ea}}+2 p_{\mathrm{A}}$ & 182.240 & -4.2 & 2.7 \\
$l+2 F-3 D+2 \Omega$ & 126.514 & 0.0 & 2.3 \\
$l+2 F+2 \Omega-18 \lambda_{\mathrm{Ve}}+16 \lambda_{\mathrm{Ea}}$ & 13.663 & -2.7 & -5.5 \\
$-l+2 F+2 \Omega+18 \lambda_{\mathrm{Ve}}-16 \lambda_{\mathrm{Ea}}$ & 13.659 & -2.7 & -5.5 \\
$l+2 F+\Omega-18 \lambda_{\mathrm{Ve}}+16 \lambda_{\mathrm{Ea}}$ & 13.635 & -0.6 & -1.1 \\
$-l+2 F+\Omega+18 \lambda_{\mathrm{Ve}}-16 \lambda_{\mathrm{Ea}}$ & 13.632 & -0.6 & 1.1 \\
$2 l+2 F+2 \Omega-18 \lambda_{\mathrm{Ve}}+16 \lambda_{\mathrm{Ea}}$ & 9.134 & -0.5 & -1.1 \\
$2 F+2 \Omega+18 \lambda_{\mathrm{Ve}}-16 \lambda_{\mathrm{Ea}}$ & 9.132 & -0.5 & 1.0 \\
\hline
\end{tabular}

Table 6. Number of coefficients of nutation $\Delta \psi$ and $\Delta \epsilon$ in common in REN and MHB for a given amplitude interval.

\begin{tabular}{lrr}
\hline \hline $\begin{array}{l}\text { Interval of amplitude } \\
\text { mas }\end{array}$ & $\begin{array}{r}\text { No. coeff. } \\
\text { in } \Delta \psi \sin \epsilon_{0}\end{array}$ & $\begin{array}{r}\text { No. coeff. } \\
\text { in } \Delta \epsilon\end{array}$ \\
\hline $10000<x$ & 0 & 0 \\
$1000<x<10000$ & 1 & 1 \\
$100<x<1000$ & 1 & 1 \\
$10<x<100$ & 7 & 5 \\
$1<x<10$ & 17 & 13 \\
$0.1<x<1$ & 40 & 35 \\
$0.01<x<0.1$ & 129 & 100 \\
$0.001<x<0.01$ & 328 & 273 \\
$0.0001<x<0.001$ & 781 & 586 \\
Total & 1304 & 1014 \\
\hline
\end{tabular}

Table 7. Number of coefficients of nutation $\Delta \psi \sin \epsilon_{0}$ and $\Delta \epsilon$ for each interval of difference $x$ in amplitude between REN and MHB.

\begin{tabular}{lrr}
\hline \hline $\begin{array}{l}\text { Interval of amplitude } \\
\text { mas }\end{array}$ & $\begin{array}{r}\text { No. coeff. } \\
\text { with diff. } x \\
\text { in } \Delta \psi \sin \epsilon_{0}\end{array}$ & $\begin{array}{r}\text { No. coeff. } \\
\text { with diff. } x \\
\text { in } \Delta \epsilon\end{array}$ \\
\hline $10<x$ & 2 & 2 \\
$1<x<10$ & 3 & 2 \\
$0.1<x<1$ & 12 & 11 \\
$0.01<x<0.1$ & 22 & 27 \\
$0.001<x<0.01$ & 58 & 57 \\
$0.0001<x<0.001$ & 139 & 191 \\
Total & 236 & 290 \\
\hline
\end{tabular}

on the nutation, it is useful to compare one by one these common coefficients. In Table 7 we show the number of coefficients for a given interval of amplitude of the difference between REN and MHB.

The 10 coefficients of $\Delta \psi$ for which the difference REN-MHB is larger than 0.5 mas are presented in Table 8, together with the absolute value of the ratio of this difference with respect to the nominal value in MHB. Globally we remark that the largest differences correspond to the largest coefficients, with arguments $\Omega, 2 F-2 D+\Omega, l^{\prime}, 2 F+2 \Omega, l, 2 \Omega$, and respective periods $18.6 \mathrm{yr}$, semi-annual (182.621d), annual (365.26d), fortnightly (13.661d), monthly (27.555d) and 9.3 yr. Moreover the ratio of the non-rigidity contribution is typically on the order of a few percent, but can reach $15 \%$ for the annual component with argument $l^{\prime}$.

The same kind of remark can be made for the largest differences in $\Delta \epsilon$ shown in Table 9. Note that the non-rigidity contribution for the semi-annual component (19.7041 mas) is very close to that of the $\Omega$ component (22.7292 mas), whereas in the case of $\Delta \psi$ it was smaller by a factor of two (39.6391 against 
Table 8. Coefficients of nutation $\Delta \psi$ with the largest difference between MHB and REN.

\begin{tabular}{lrrrrr}
\hline \hline Argument & $\begin{array}{r}\text { Period } \\
\text { days }\end{array}$ & $\begin{array}{r}\text { Sine } \\
\text { mas }\end{array}$ & $\begin{array}{r}\text { Cosine } \\
\text { mas }\end{array}$ & $\begin{array}{r}\text { total } \\
\text { mas }\end{array}$ & $\begin{array}{r}\text { radio } \\
\text { diff./amplitude }\end{array}$ \\
\hline$\Omega$ & 6798.384 & 74.1760 & 2.9560 & 74.2349 & 0.0043 \\
$2 \Omega$ & 3399.192 & -1.5742 & -0.0757 & 1.5760 & 0.0076 \\
$l^{\prime}$ & 365.260 & 22.0841 & 1.1817 & 22.1157 & 0.1498 \\
$2 F-2 D+\Omega$ & 182.621 & -39.6154 & -1.3696 & 39.6391 & 0.0301 \\
$l^{\prime}+2 F-2 D+2 \Omega$ & 121.749 & -1.7443 & -0.0524 & 1.7451 & 0.0338 \\
$-l+2 D$ & 31.812 & 0.7410 & -0.0168 & 0.7412 & 0.0472 \\
$l$ & 27.555 & 3.3482 & -0.0872 & 3.3493 & 0.0471 \\
$2 F+2 \Omega$ & 13.661 & -6.1301 & 0.2796 & 6.1365 & 0.0270 \\
$2 F+\Omega$ & 13.633 & -0.8772 & 0.0380 & 0.8780 & 0.0227 \\
$l+2 F+2 \Omega$ & 9.133 & -0.5633 & 0.0816 & 0.5692 & 0.0189 \\
\hline
\end{tabular}

Table 9. Coefficients of nutation $\Delta \epsilon$ with the largest difference between MHB and REN.

\begin{tabular}{lrrrrr}
\hline \hline Argument & $\begin{array}{r}\text { Period } \\
\text { days }\end{array}$ & $\begin{array}{r}\text { Sine } \\
\text { mas }\end{array}$ & $\begin{array}{r}\text { Cosine } \\
\text { mas }\end{array}$ & $\begin{array}{r}\text { total } \\
\text { mas }\end{array}$ & $\begin{array}{r}\text { radio } \\
\text { diff./amplitude }\end{array}$ \\
\hline$\Omega$ & 6798.384 & 1.5408 & -22.6769 & 22.7292 & 0.0025 \\
$2 \Omega$ & 3399.192 & -0.0323 & 0.5877 & 0.5886 & 0.0066 \\
$l^{\prime}$ & 365.260 & -0.1924 & 7.5220 & 7.5245 & 1.0182 \\
$2 F-2 D+\Omega$ & 182.621 & -0.4587 & 19.6988 & 19.7041 & 0.0344 \\
$l^{\prime}+2 F-2 D+2 \Omega$ & 121.749 & -0.0174 & 0.8187 & 0.8189 & 0.0365 \\
$l$ & 27.555 & 0.0358 & 0.2975 & 0.2996 & 0.4433 \\
$2 F+2 \Omega$ & 13.661 & 0.1374 & 2.9250 & 2.9282 & 0.0299 \\
$2 F+\Omega$ & 13.633 & 0.0318 & 0.6603 & 0.6611 & 0.0329 \\
$l+2 F+2 \Omega$ & 9.133 & 0.0367 & 0.2928 & 0.2951 & 0.0229 \\
\hline
\end{tabular}

74.2349). Note also the specifity of the annual term $\left(l^{\prime}\right)$ whose ratio is larger than 1: the geophysical contributions are much larger for this sole term than the nominal value for a rigid Earth model. The reason is the proximity of a resonance associated with the (retrograde) free core nutation (period of about 430 days)

\section{Long periodic coefficients and truncation level}

In this section we carry out complementary studies to answer the following two questions:

- what are the influences of the very long periodic coefficients on the global nutation and to what extent can their effect be simply approximated by polynomials?

- what are the cumulative effects of the smallest coefficients and to what extent (of amplitude) can they be ignored in comparison with the accuracy and the precision of present observations of the nutation, noticeably through VLBI measurements?

\subsection{Long periodic coefficients}

A substantial number of terms in both $\Delta \psi$ and $\Delta \epsilon$ have such a large period that, for a rather long time interval (e.g., one century), their effect can be assimilated to a secular trend. Therefore it looks worthy studying how the global nutation effect given by these coefficients could be fitted by a single polynomial. Note that for all 142 coefficients in $\Delta \psi$ and for 94 coefficients in $\Delta \epsilon$ with period longer than 18.6 years (corresponding to the largest nutation term with argument $\Omega$ ) their amplitude in REN and in MHB are exactly the same, meaning that the effect of nonrigidity is negligible.

Figure 1 shows four curves for $\Delta \psi$ corresponding to a given threshold for the period. Thus the bold curve represents the global nutation $\Delta \psi$ by keeping the 142 coefficients with periods laonger than 6798 days (18.6 years). We observe that this

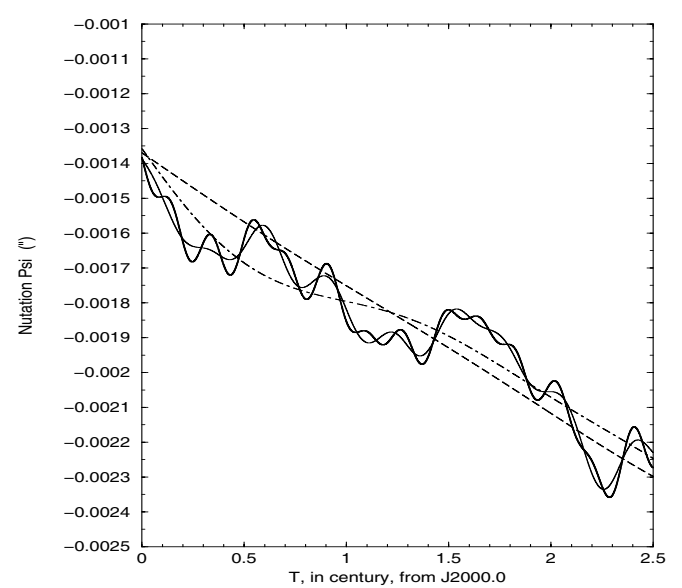

Fig. 1. Curves showing the global nutation $\Delta \psi$ from MHB when keeping the terms with period larger than the leading $18.6 \mathrm{yr}$ component (bold), larger than $10000 \mathrm{~d}$ (close to the first one), larger to $50000 \mathrm{~d}$ (lightly rounded), and larger than $100000 \mathrm{~d}$ (close to a straight line).

curve shows some dominant oscillations that are still present when keeping the 125 coefficients with $T>10000 \mathrm{~d}$ (second curve very close to the first one). By contrast, the third and fourth curves obtained respectively by keeping the 48 coefficents with $T>50000 \mathrm{~d}$ and the 32 coefficients with $T>100000 \mathrm{~d}$ can be easily assimilated to a low-order polynomial and a straight line.

Figure 3 shows the curve obtained by keeping all the terms with $T>50000 \mathrm{~d}$, together with the curve obtained by fitting a cubic regression. The post-fit residuals are shown in Fig. 5 together with the post-fit residuals after fitting with a fourthorder polynomials and a fifth order one. The fitting polynomials are $P_{3}(T)=-1.39-0.7192 T+0.4052 T^{2}-0.1048 T^{3}$, $P_{4}(T)=-1.335-1.156 T+1.192 T^{2}-5.942 T^{3}+0.098 T^{4}$, and $P_{5}(T)=-1.341-1.088 T+1.001 T^{2}-3.907 T^{3}+0.006 T^{4}+0.15 T^{5}$ 


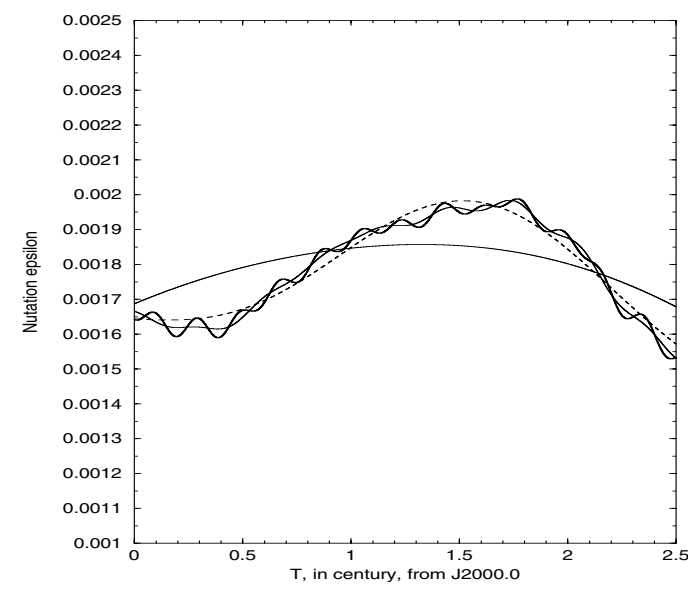

Fig. 2. Curve showing the global nutation $\Delta \epsilon$ from MHB when keeping the terms with period larger than the leading $18.6 \mathrm{yr}$ component (bold), larger than $10000 \mathrm{~d}$ (close to the first one), larger to $50000 \mathrm{~d}$ (dashed, lightly rounded), and larger than $100000 \mathrm{~d}$ (well-separated from the others).

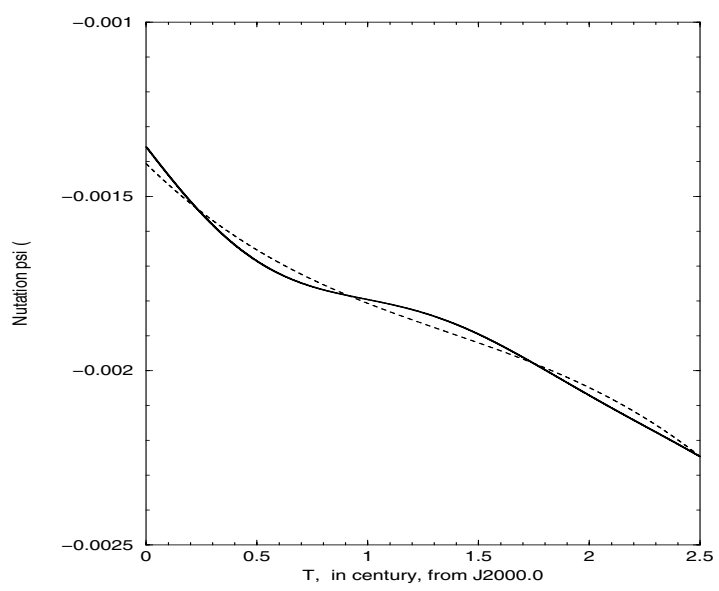

Fig. 3. Curve showing the global nutation $\Delta \psi$ from MHB when keeping only the terms with period larger than $50000 \mathrm{~d}$ (bold) with the curve obtained through cubic regression.

where $T$ is expressed in Julian centuries from $\mathrm{J} 2000.0$ and the unit for $P_{i}(t)$ is the mas.

We can also observe that, although the original signal is varying secularly with an amplitude of 1 mas for 250 years, the residuals in Fig. 5 remain in a 0.08 mas interval (cubic regression) and a 0.05 mas one (4th-order regression).

We have done the same kind of study for $\Delta \epsilon$ which variability for a 250 years time span from J2000.0 is of the order of 0.5 mas (peak to peak). 94 coefficients are retained for $T>6798 \mathrm{~d}$, 77 for $T>10000 \mathrm{~d}, 33$ for $T>50000 \mathrm{~d}$ and 20 ones for $T>100000 \mathrm{~d}$. Note that, whatever the threshold adopted for the period, the signal (Fig. 2) does not present a net secular trend as for $\Delta \psi$.

The global value of $\Delta \epsilon$ by keeping only the terms with $T>50000 \mathrm{~d}$ is shown in Fig. 4 with the curve representing the fitting polynomial after cubic regression. The residuals, after adopting this regression and two other ones of 4th and 5th order, are shown in Fig. 6. Notice that here the 5th order polynomial regression leads to significantly smaller residuals than the two other ones, i.e., between \pm 0.01 mas. The fitting polynomials are $P_{3}(T)=1.624-0.0424 T+0.4452 T^{2}-0.1803 T^{3}$,

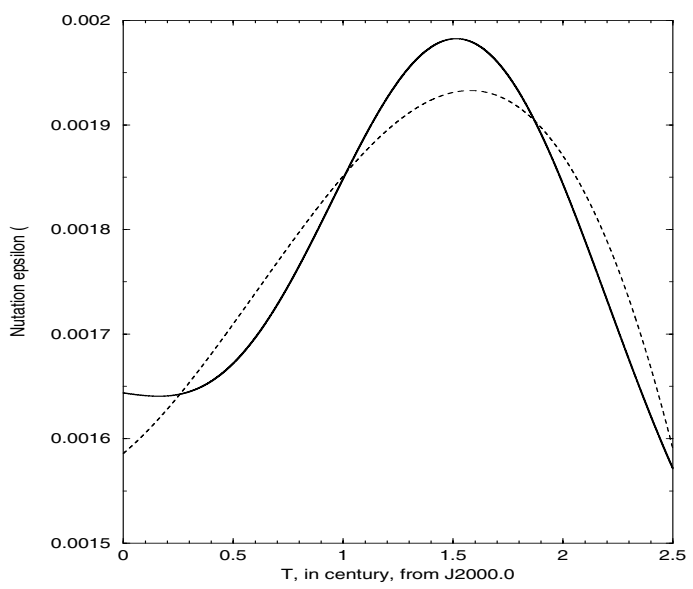

Fig. 4. Curve showing the global nutation $\Delta \epsilon$ from MHB when keeping only the terms with period larger than $50000 \mathrm{~d}$ (bold) with the curve obtained through cubic regression.

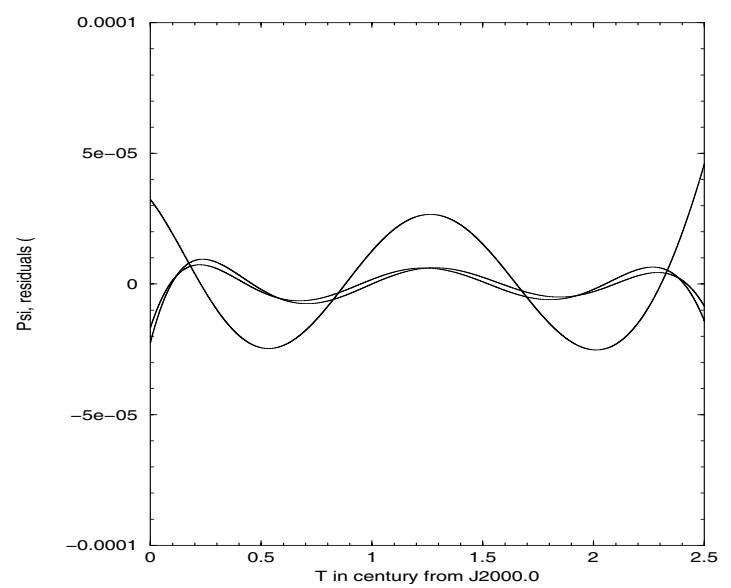

Fig. 5. Curves showing the residuals after the cubic regression for $\Delta \psi$ in Fig. 3, after polynomial regression with order 4 and order 5 (close curves).

$P_{4}(T)=1.687-0.545 T+1.35 T^{2}-0.7432 T^{3}+0.1126 T^{4}$, $P_{5}(T)=1.649-0.8261 T+0.05515 T^{2}+0.6378 T^{3}-0.5089 T^{4}$.

Therefore we can conclude that, for a time span on the scale of one century, a large number of long periodic terms in the nutation series might be advantageously replaced by a polynomial of order 3,4 , or 5 according to the degree of approximation required. Notice that the polynomials $P_{i}$ have been defined for a 250-year time span between 2000 and 2250, and their values could be noticeably affected by the time span considered.

Following these results we must emphasize the ambiguity consisting in assimilating the linear trend due to long-period nutations with precession. In the case of the general precession in longitude, roughly $50^{\prime \prime} / y$, a linear trend of about 1 mas/cy amplitude as observed in the expressions $P_{i}$ above corresponds to a relative $2 \times 10^{-7}$ ratio.

\subsection{Truncation level vs. VLBI}

In the following paragraph, we use the IERS EOP C 04 data set (Gambis 2006, referred to as C04 in the following) provided by the International Earth Rotation and Reference Systems Service (IERS) as observational reference. This data is obtained by a weighted combination of individual VLBI data sets provided by 


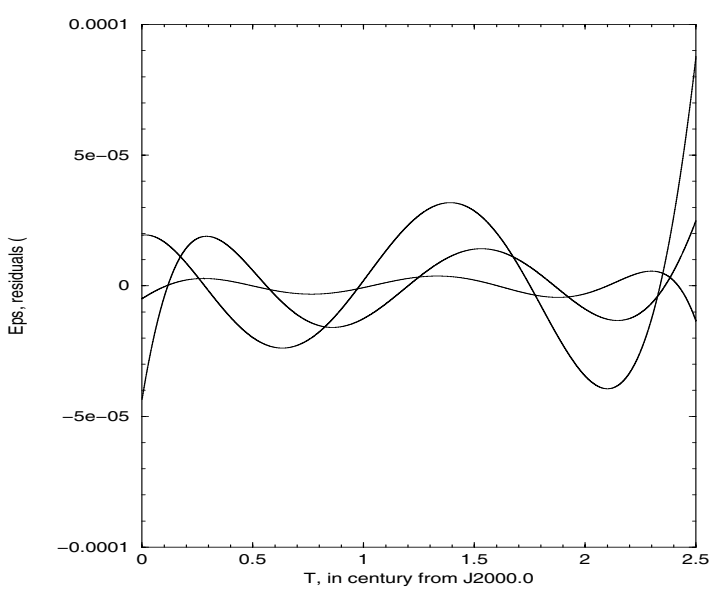

Fig. 6. Curves showing the residuals after the cubic regression for $\Delta \epsilon$ in Fig. 4, after polynomial regression with 4th. and 5th. order (close curves).

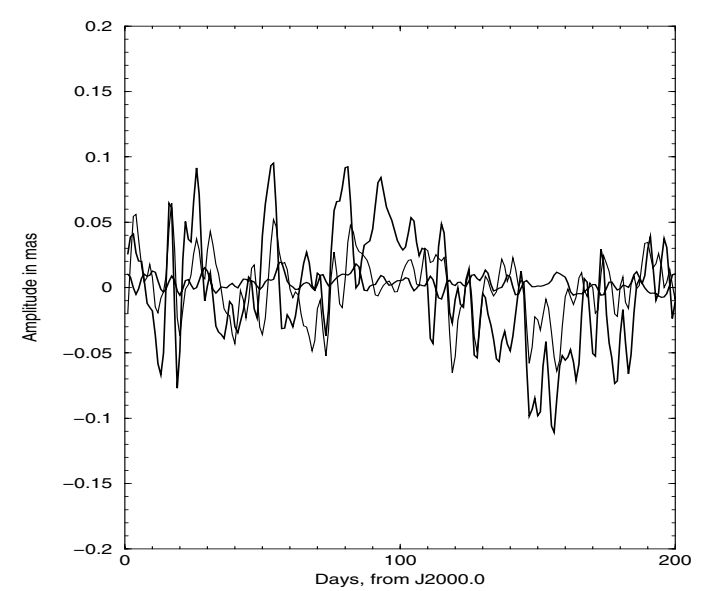

Fig. 7. Curve showing the global nutation $\Delta \psi$ in MHB obtained by keeping only the coefficients smaller than $10 \mu$ as, $5 \mu$ as, and $1 \mu$ as easily identified by decreasing order of amplitude.

various VLBI analysis centers. The use of another individual or combined VLBI data set would not lead to significantly different results. To efficiently compare the nutation models with their observational counterparts, one has to remove any signal that is not linked to the nutation modeling. This is the case of the retrograde free-core nutation (RFCN) whose physical aspects are briefly discussed in the last section of this paper. In terms of signal processing, the RFCN shows a quasi periodic retrograde oscillation of period between 430 and 460 days and of variable and unpredictable amplitude ( $200 \mu$ as) and phase. Although unpredictable, the RFCN can be removed from VLBI observations using a least-square fit of a sinusoid with fixed period (e.g., at the MHB value of 430.21 days) but unknown phase and amplitude. A 2-yr sliding window shifted by one year accounts for the time variability of the amplitude and the phase.

Then we adopt three truncature thresholds both for $\Delta \psi$ (Fig. 7) and for $\Delta \epsilon$ (Fig. 8): these are $10 \mu$ as, $5 \mu$ as, and $1 \mu$ as. Adopting a $10 \mu$ as threshold leads to ignoring a signal with roughly \pm 0.1 mas (peak to peak, see Figs. 7-8), which is quite big in comparison with (i) the non-rigid Earth contributions to the leading coefficients, and (ii) the accuracy of observational data (0.1 mas). By contrast, ignoring all the coefficients smaller

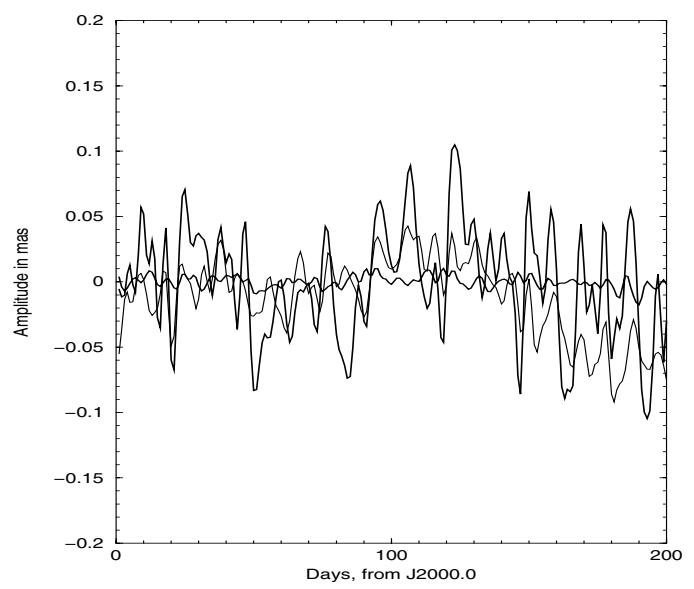

Fig. 8. Curve showing the global nutation $\Delta \epsilon$ in MHB obtained by keeping only the coefficients smaller than $10 \mu \mathrm{as}, 5 \mu \mathrm{as}$, and $1 \mu \mathrm{as}$ easily identified by decreasing order of amplitude.

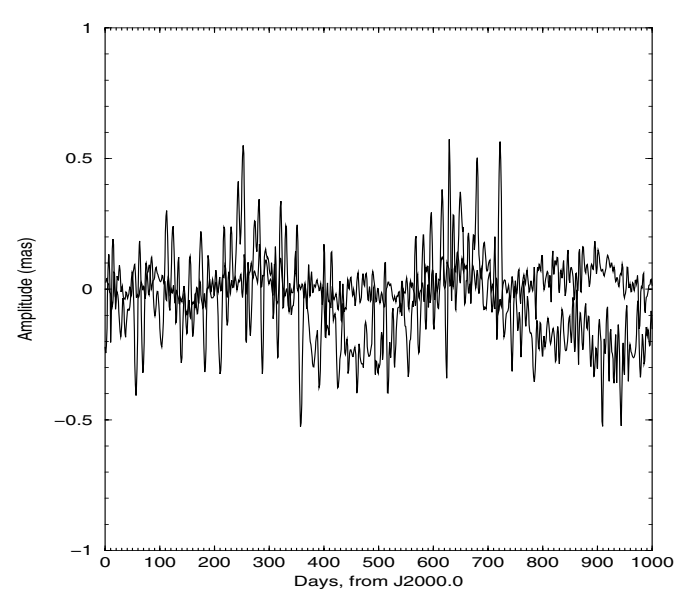

Fig. 9. Curve showing the global nutation $\Delta \psi$ in MHB obtained by keeping only the coefficients smaller than $10 \mu$ as, (very flat) in comparison with the residuals between MHB and observational data (large amplitude).

than $1 \mu$ as does not significantly affect the difference between observations and model, as can be seen in Figs. 9 and 10 for $\Delta \psi$ and $\Delta \epsilon$, respectively. The curves with large amplitudes represent the differences between the observed nutation angles as given by the $\mathrm{C} 04$ data set once the RFCN oscillation is removed, and the MHB model, whereas the flat curves (bold) represent the global nutation given by the coefficients with amplitudes smaller than $1 \mu$ as. While the first ones have amplitudes ranging in the interval \pm 0.5 mas in $\Delta \psi$ and in $\Delta \epsilon$ (peak to peak), the amplitudes of the second ones do not exceed 0.15 mas.

Finally we calculate the residuals $\delta \psi$ and $\delta \epsilon$ obtained by subtracting the truncated MHB series with a given threshold from the $\mathrm{C} 04$ series (i.e., we do not take into account the coefficients under this threshold). The results are presented in Table 10. We consider two cases, one with the original C04 series, the other with the $\mathrm{C} 04$ series from which the $\mathrm{RFCN}$ is removed. We note that, as long as we neglect the coefficients in MHB smaller than $5 \mu$ as, the residuals are not significantly affected by the truncation. Nevertheless the situation worsens significantly when the truncation threshold exceeds $10 \mu$ as both for $\delta \psi$ and $\delta \epsilon$. In conclusion we can assert that ignoring the 781 coefficients for $\Delta \psi$ 
Table 10. rms of the global nutations $\Delta \psi$ and $\Delta \epsilon$ when ignoring coefficients under a given threshold, together with the corresponding residuals of the $\mathrm{C} 04$ minus MHB both by taking or not the RFCN correction into account.

\begin{tabular}{|c|c|c|c|c|c|c|}
\hline Threshold & $\begin{array}{r}\delta \psi \mathrm{rms} \\
\text { ignored coeff. }\end{array}$ & $\begin{array}{r}\delta \psi \mathrm{rms} \\
\mathrm{C} 04-\mathrm{MHB}\end{array}$ & $\begin{array}{r}\delta \psi \mathrm{rms} \\
\mathrm{C} 04-\mathrm{RFCN}-\mathrm{MHB}\end{array}$ & $\begin{array}{r}\delta \epsilon \mathrm{rms} \\
\text { ignored coeff. }\end{array}$ & $\begin{array}{r}\delta \epsilon \mathrm{rms} \\
\mathrm{C} 04-\mathrm{MHB}\end{array}$ & $\begin{array}{r}\delta \epsilon \mathrm{rms} \\
\text { C04-RFCN-MHB }\end{array}$ \\
\hline$\mu$ as & mas & mas & mas & mas & mas & mas \\
\hline 0 & 0.0000 & 0.4551 & 0.3887 & 0.0000 & 0.2089 & 0.1792 \\
\hline 0.5 & 0.0038 & 0.4552 & 0.3885 & 0.0034 & 0.2088 & 0.1789 \\
\hline 1.0 & 0.0079 & 0.4551 & 0.3884 & 0.0066 & 0.2101 & 0.1804 \\
\hline 5.0 & 0.0356 & 0.4559 & 0.3948 & 0.0289 & 0.2106 & 0.1828 \\
\hline 10.0 & 0.0591 & 0.4577 & 0.3879 & 0.0452 & 0.2126 & 0.1837 \\
\hline 50.0 & 0.2426 & 0.4663 & 0.3818 & 0.1659 & 0.2696 & 0.2456 \\
\hline 100.0 & 0.4121 & 0.5355 & 0.4677 & 0.2714 & 0.3911 & 0.3684 \\
\hline
\end{tabular}

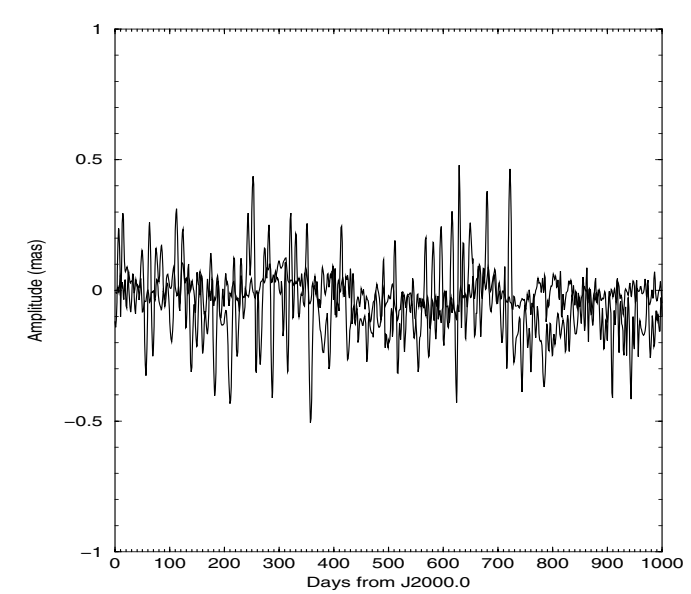

Fig. 10. Curve showing the global nutation $\Delta \epsilon$ in MHB obtained by keeping only the coefficients smaller than $10 \mu \mathrm{as}$, (very flat) in comparison with the residuals between MHB and observational data (large amplitude).

and 586 for $\Delta \epsilon$ with amplitude smaller than $1 \mu$ as in MHB (see Table 6) will not influence the quality of interpretation of observational data.

\section{Concluding remarks: a word on the remaining variance}

In this paper we have described in detail the term-by-term differences between the REN nutation series for a rigid Earth model and the MHB nutation series for a non-rigid Earth model. After listing abundantly the characteristics of these series and studying the effects of their truncation to a limited number of terms, we pointed out the effects of non-rigidity on the leading coefficients. We think that all these studies are useful for the common user of nutation series.

It was mentioned earlier that the VLBI-derived nutation angles against the MHB model show differences of about $200 \mu$ as in rms. This remaining variance points out limitations in our knowledge of the Earth's interior and the interactions between solid Earth and geophysical fluid layers, the latter effects being typically around $80 \mu \mathrm{as}$, i.e. half the full variance (see e.g., Dehant et al. 2003, and references therein). Other major difficulties deal with geodetic VLBI measurements that present very high sensitivity to instrumental capabilities and to observational and analysis strategies. This includes the influence of the VLBI network geometry on Earth orientation determination (Feissel-Vernier et al. 2004; Lambert \& Gontier 2006), the troposphere gradient modeling (MacMillan \& Ma 1999), the constraints on the radio- source coordinates to free the nutation offsets from reference-frame effects (Ma et al. 1998; Feissel-Vernier et al. 2005), and some software issues. Moreover, an unpredictable signal shows up in the VLBI residuals at the level of $200 \mu \mathrm{as}$, which is due to the excitation of the Earth's free rotational mode associated with the ellipsoidal liquid core rotating inside the visco-elastic mantle and known as the retrograde free core nutation (RFCN). This free mode is thought to be excited by the external fluid layers. However, although the amplitude of the RFCN oscillation is globally explained by the diurnal atmospheric pressure variations on the crust (see, e.g., Vondrák \& Ron 2006), its amplitude and phase variability in time, noticed in VLBI observations (see, e.g., Herring et al. 2002), is still under investigation, a fact partly due to a lack of reliable meteorological data in the diurnal band (Lambert 2006).

In the next years, a strong challenge will be to describe the Earth rotation in a consistent relativistic framework. It is well known that the notion of rigid body is generally ill-defined in Relativity, even in the first post-Newtonian approximation (Thorne \& Gürsel 1983). Nevertheless one may find some attempts in the literature to extend rigid Earth nutation from a Newtonian to relativistic framework (Brumberg 1972; Damour et al. 1993; Klioner 1996; Xu et al. 2004). Usually to get this extension, one has to apply a four-dimensional transformation between geocentric and barycentric quantities on the right-hand side of the Newtonian equations of motion. This approach is not satisfactory and an alternative exists. By using the approximation of rigidly rotating multipoles (Klioner et al. 2003), it is then possible to derive a set of equations that describes the Earth rotation with respect to the Geocentric Celestial Reference System. This way has to be carefully investigated as soon as possible in order to fully understand the influence of Relativity on Earth rotation and the impact on high-precision analysis of Earth's orientation observational data.

\section{References}

Bretagnon, P., Rocher, P., \& Simon, J.-L. 1997, A\&A, 319, 305 Brumberg, V. A. 1972, Relativistic Celestial Mechanics (Moscow: Nauka) Damour, T., Soffel, M., \& Xu, C. 1993, Phys. Rev. D, 47, 3124

Dehant, V., Feissel-Vernier, M., de Viron, et al. 2003, J. Geophys. Res., 108(B5) Feissel-Vernier, M., Ray, J., Altamimi, Z., et al. 2004, in International VLBI Service for Geodesy and Astrometry (IVS) 2004 General Meeting Proceedings, ed. N. R. Vandenberg, \& K. D. Baver, NASA/CP-2004212255, 22

Feissel-Vernier, M., Ma, C., Gontier, A.-M., \& Barache, C. 2005, A\&A, 438, 1141

Folgueira, M., Souchay, J., \& Kinoshita, H. 1998a, Cel. Mech. Dyn. Astrom., 69,373 
Folgueira, M., Souchay, J., \& Kinoshita, H. 1998b, Cel. Mech. Dyn. Astrom., 70,147

Gambis, D. 2006, private communication

Kinoshita, H. 1972, Publ. Astron. Soc. Japan, 24, 423

Kinoshita, H. 1977, Cel. Mech. Dyn. Astrom., 15, 277

Kinoshita, H., \& Souchay, J. 1990, Cel. Mech. Dyn. Astron., 48, 187

Klioner, S. A. 1996, Dynamics, Ephemerides, and Astrometry of the Solar System, IAU Symp., 172, 309

Klioner, S. A., Soffel, M., Xu, C., \& Wu, X. 2003, Journées 2001 - systèmes de référence spatio-temporels. Influence of geophysics, time and space reference frames on Earth rotation studies, Brussels, 24-26 September 2001, ed. N. Capitaine, Observatoire de Paris, Systèmes de Référence TempsEspace, UMR 8630/CNRS; Observatoire Royal de Belgique, 13, 232

Lambert, S. B. 2006, A\&A, 457, 717

Lambert, S. B., \& Gontier, A.-M. 2006, in International VLBI Service for Geodesy and Astrometry (IVS) 2006 General Meeting Proceedings, ed. D. Behrend, \& K. D. Baver, NASA/CP-2006-214140, 264
Lambert, S. B., \& Mathews, P. M. 2006, A\&A, 453, 363

Ma, C., Arias, E. F., Eubanks, T. M., et al. 1998, AJ, 116, 516

MacMillan, D. S., \& Ma, C. 1999, Geophys. Res. Lett., 267, 919

Mathews, P. M., Buffett, B. A., Herring, T. A., \& Shapiro, I. I. 1991, J. Geophys. Res., 96(B5), 8243

Mathews, P. M., Herring, T. A., \& Buffett, B. A. 2002, J. Geophys. Res., 107(B4)

Roosbeek, F., \& Dehant, V. 1998, Celest. Mech. Dyn. Astron., 70(4), 215

Souchay, J. A. J. 1998, 116, 503

Souchay, J., \& Kinoshita, H. 1996, A\&A, 312, 1017

Souchay, J., \& Kinoshita, H. 1997, A\&A, 318, 639

Souchay, J., Loysel, B., Kinoshita, H., Folgueira, M. 1999, A\&AS, 135, 111

Thorne, K. S., \& Gürsel, Y. 1983, MNRAS, 205, 809

Vondrák, J., \& Ron, C. 2006, Acta Geodyn. Geomater., 3(143), 53

Wahr, J. M. 1981, Geophys. J.R. Astron. Soc., 64, 705

Xu, C., Tao, J.-H., \& Wu, X. 2004, Phys. Rev. D, 69, 024003 